\title{
Guest Editorial: Special Issue: Computer Vision and Pattern Recognition (DAGM GCPR 2019)
}

\author{
Simone Frintrop ${ }^{1} \cdot$ Gernot A. Fink ${ }^{2} \cdot$ Xiaoyi Jiang ${ }^{3}$
}

Accepted: 17 July 2021 / Published online: 27 August 2021

(c) The Author(s) 2021

This special issue consists of 4 papers covering several pattern recognition topics selected from the DAGM GCPR conference (German Conference on Pattern Recognition) held in Dortmund, Germany, September 10-13, 2019. It was the 41st edition of an international conference series organized by DAGM (The German Association for Pattern Recognition), which was run under the name DAGM Symposium for Pattern Recognition until 2012 und German Conference on Pattern Recognition afterwards.

A selection of 5 papers ranked highest by an evaluation committee during the conference were invited by us, the chairs of DAGM GCPR 2019, to submit extended manuscripts to this special issue. These submissions underwent an additional rigorous peer-review process and 4 were finally accepted for this special issue. Let us briefly sketch the idea of these papers.

In their paper "Learning Extremal Representations with Deep Archetypal Analysis" Sebastian Mathias Keller, Maxim Samarin, Fabricio Arend Torres, Mario Wieser, and Volker Roth address the problem of simultaneously finding an archetypal representation of a data distribution and a suitable feature space that allows a good representation by a convex hull spanned by the archetypes. To this end, they propose a novel framework in which a generative model based on a variational autoencoder can be trained end-to-end allowing the incorporation of side information about the task considered.

In the paper entitled "Tracking by Deblatting" Denys Rozumnyi, Jan Kotera, Filip Šroubek, and Jiří Matas propose a novel method for tracking fast-moving objects in videos that travel considerable distances between frames. The

Simone Frintrop

simone.frintrop@uni-hamburg.de

1 University of Hamburg, Hamburg, Germany

2 TU Dortmund, Dortmund, Germany

3 University of Münster, Münster, Germany approach is based on the estimation of the motion blur that such objects undergo. The procedure solves two intertwined inverse problems, namely blind deblurring and image matting, which the authors then call deblatting. Via a series of post-processing operations, continuous trajectories are generated for the tracked objects.

Patrick Knöbelreiter and Thomas Pock present their work on "Learned Collaborative Stereo Refinement", which is a learning-based method to denoise and refine disparity maps. They address the problem with a hierarchical variational network, which allows to exploit both the power of deep learning and the interpretability of variational methods. The result is a simple, effective, and interpretable refinement approach for disparity maps.

The paper "Entrack: Probabilistic Spherical Regression with Entropy Regularization for Fiber Tractography" by Viktor Wegmayr and Joachim Buhmann presents a novel probabilistic fiber tractography algorithm. It combines a probabilistic model for predicting local fiber orientation with a machine learning strategy that is used to predict fiber orientations based on the modelled posterior and the data.

As guest editors, we would like to thank all authors for their valuable contributions to this special issue. We also thank the reviewers, who made helpful suggestions that further improved the quality of the papers, and the editorial staff of IJCV for their support. All of them gave their best to contribute to this issue, despite the challenging Corona pandemic time with many of us working in home office, some handling the care and home schooling of their kids and dealing with other difficult conditions. It is thanks to the effort of everyone that the quality of the contributions was not affected in any way by the pandemic. With vaccination programs proceeding fast, we are optimistic that conferences such as DAGM GCPR and other events will be possible again in a near future. 
Funding Open Access funding enabled and organized by Projekt DEAL.

Open Access This article is licensed under a Creative Commons Attribution 4.0 International License, which permits use, sharing, adaptation, distribution and reproduction in any medium or format, as long as you give appropriate credit to the original author(s) and the source, provide a link to the Creative Commons licence, and indicate if changes were made. The images or other third party material in this article are included in the article's Creative Commons licence, unless indicated otherwise in a credit line to the material. If material is not included in the article's Creative Commons licence and your intended use is not permitted by statutory regulation or exceeds the permitted use, you will need to obtain permission directly from the copyright holder. To view a copy of this licence, visit http://creativecommons.org/licenses/by/4.0/.

Publisher's Note Springer Nature remains neutral with regard to jurisdictional claims in published maps and institutional affiliations. 